\title{
High-throughput intrusion detection system with parallel pattern matching
}

\author{
Yi-Mao Hsiao ${ }^{\text {a) }}$, Ming-Jen Chen, Yuan-Sun Chu, \\ and Chung-Hsun Huang \\ Institute of Electrical Engineering, National Chung Cheng University, \\ 168 University Road, Minshiung, Chia-Yi, Taiwan 62102, R.O.C. \\ a)93mowmow@vlsi.ee.ccu.edu.tw
}

\begin{abstract}
This paper proposes a high-throughput intrusion detection system (IDS) with a bloom filter-based header comparison and parallel pattern matching for the packet content. The parallel pattern matching is a two parallel sequence comparison architecture that compares the packet content with the Snort rules. The proposed hardware IDS not only performs high throughput, but also reduces the rules memory size. As shown in post-layout simulation of the implemented application-specific integrated circuit (ASIC), the speed reaches $453 \mathrm{MHz}$ that performs $7.2 \mathrm{Gbps}$ system throughput to deal with the traffic requirement of edge speed in end user network. With $8 \mathrm{MB}$ offchip SRAM, the system supports 4,020 Snort rules that the pattern number is enough for intruder signature.
\end{abstract}

Keywords: IDS, Snort, ASIC

Classification: Integrated circuits

\section{References}

[1] T. AbuHmed, A. Mohaisen, and D. H. Nyang, "A Survey on Deep Packet Inspection for Intrusion Detection Systems," Magazine of Korea Telecommunication Society, vol. 24, no. 11, pp. 25-36, Nov. 2007.

[2] I. Sourdis, V. Dimopoulos, D. Pnevmatikatos, and S. Vassiliadis, "Packet Pre-filtering for Network Intrusion Detection," ACM/IEEE Symp. Architecture for Networking and Communications Systems, pp. 183-192, Dec. 2006.

[3] E. Azimi, M. B. Ghaznavi-Ghoushch, and A. M. Rahmani, "Implementation of simple SNORT processor for efficient Intrusion Detection systems," IEEE Int. Conf. Intelligent Computing and Intelligent Systems, pp. 533537, Nov. 2009.

[4] C.-H. Lin and S.-C. Chang, "Efficient pattern matching algorithm for memory architecture," IEEE Trans. Very Large Scale Integr. (VLSI) Syst., vol. 19, no. 1, pp. 33-41, Jan. 2011.

[5] A. G. Alagu Priya and H. Lim, "Hierarchical packet classification using a Bloom filter and rule-priority tries," Computer Communications, pp. 1215-1226, March 2010.

[6] H. Yu and R. Mahapatra, "A Memory-Efficient Hashing by MultiPredicate Bloom Filters for Packet Classification," Proc. IEEE Infocom, pp. 1795-1803, April 2008. 
[7] O. Yigit, "Substitute-dbm," 1990. [Online] http://search.cpan.org/src/ NWCLARK/perl-5.8.4/ext/SDBM File/sdbm

\section{Introduction}

With the development of the Internet, viruses and malicious software are spread quickly. The IDS is the commonly used network security infrastructure in recent years such as the SNORT software. The main problem with software IDS is their inadaptability with increasing rates from the network. To resolve this problem, a hardware IDS system provides more parallel processing capability and effectiveness for the requirement of the current network environment.

AbuHmed surveyed the deep packet inspection for IDS. He indicated the design challenge is the design of high throughput hardware architecture to process intruder signature for the traffic requirement of speed in end user network as $1 \mathrm{Gbps}$ and core speed on network as 10 to 40 Gbps [1]. Sourdis proposed a packet pre-filtering approach that selected a small portion from the rules for IDS [2]. This pruned set of rules may apply to a packet, a second-stage, and full-match engine. They implemented the pre-filtering approach as the reconfigurable architecture to minimize the processing. Azimi proposed a hardware implementation of the basic instructions of the SNORT processor for IDS [3]. The SNORT processor was a hardware accelerator of IDS in a 1 Gbs data rate network. Among the hardware approaches, memorybased architecture is used to accommodate the increasing number of attack patterns and to meet the throughput requirement of networks. The Snort rules were implemented with a finite state machine (FSM). Lin proposed a memory-efficient pattern-matching algorithm with FSM hardware design that markedly reduced the memory requirement for Snort rule sets [4]. The bloom filter is an efficient data structure for membership queries and is widely used in the network applications. Priya proposed a hierarchical tree structure to improve system throughput and memory usage for the bloom filter [5]. Yu proposed the multi-predicate bloom filtered hash architecture that used parallel bloom filters [6]. The system contained on-chip and off-chip memory. Their architecture reduced the on-chip memory size and achieved efficient memory access for off-chip memory.

In this paper, we propose a high-throughput IDS with a bloom filter-based searching and a parallel pattern-matching architecture. The bloom filter is the search engine for the packet header and the parallel pattern-matching architecture to compare the payload of the packet. The parallel pattern matching is a two parallel sequence comparison architecture that compares the packet content with the Snort rules. The system also monitors the dedicated IP addresses and then tells the firewall to protect denial of service attack. We implement the IDS into an ASIC. The ASIC achieves 7.2 Gbps throughput to manage the requirement of line speed from the network. The 
system supports 4,020 Snort rules, which are stored in the off-chip memory.

\section{Proposed IDS system}

The proposed IDS system detects virus and malicious software attacks on the Internet and then notices the firewall to protect the users in the end system. When the packets are received, the system uses the pre-filter to divide the packets of information into the header and the packet content payload. The header information is assessed by the header matching systems. If compared to the packet header, this header is the corresponding rule out from the memory. The content information is assessed by the content matching system. The IDS manages the information within the content to perform rule matching. Then, the system determines whether there is a virus or malicious. The hash function is widely used for search algorithms since it performs time complexity as $O(1)$ in searching. The bloom filters is a hash-based architecture with efficient probability. We design the bloom filter as the search engine. The sdbm [7] is used as the input string hash function for high-speed searching since the computing time of the sdbm is short. The hash function is as follows:

$$
\operatorname{hash}(i)=\operatorname{hash}(i-1) * 65599+\operatorname{str}[i]
$$

Selecting the bloom filter as the search engine offers two advantages. First, the hash function searches the location of the data in a short period. Second, the bloom filter reduces the data storage memory size. Searching is active by the first hash function operation. The result is divided into two parts after the operation. One part is used for the bloom filter to write data, and the other is that the system stores the data into the memory. The disadvantage of the hash function is the collisions. We design the method to resolve the collision problem. If the bit value is mapped to " 1 ", the search filters in a single dimension correspond to the next bit, and the value is written as "1". The bloom filter design is shown more detail in the next section.

\section{System architecture}

The proposed IDS hardware architecture contains a header matching system, a parallel content compare system, and off-chip memory, as shown in Fig. 1(a). A packet is composed of a header and payload. The received packet includes the source IP, source port, destination IP and destination port in the header information. These data are compared by the header address search system, as shown in Fig. 1 (b). When compared to the successful detection, the parallel content compare processes the content block. If the system detects no problems, the IDS stops the matching system and waits for the next data input. When comparing the payload, the content is sent to the parallel compare system. The header of the content and the rule is the same if the parity of the even part and the odd part signal is bit 1. The IDS sends out match signals success of this virus packet. 


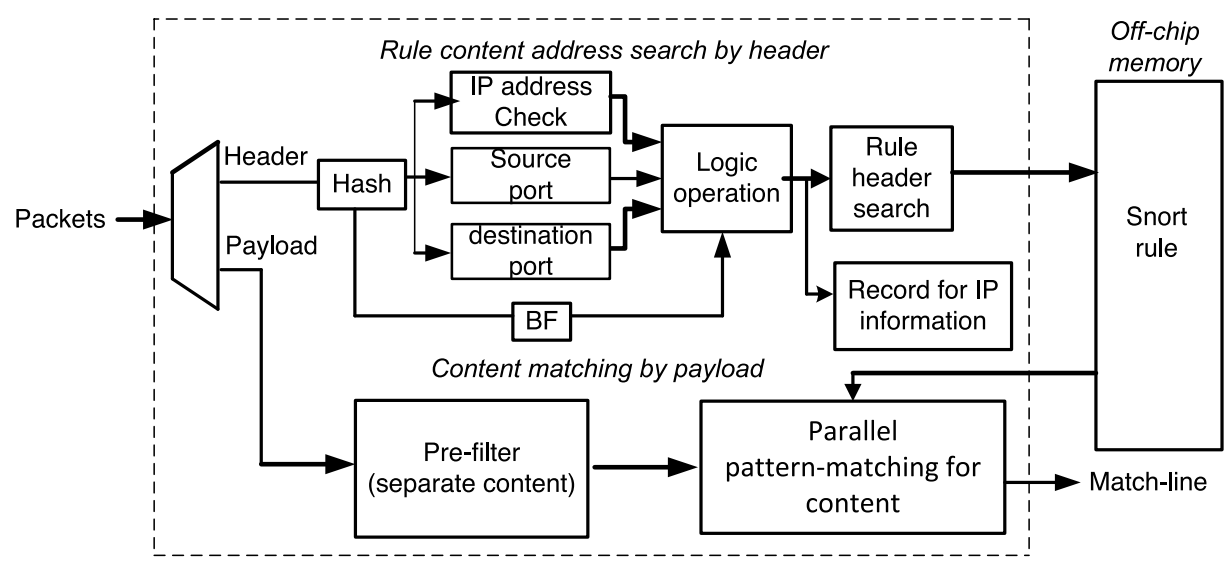

(a) The System Architecture of IDS
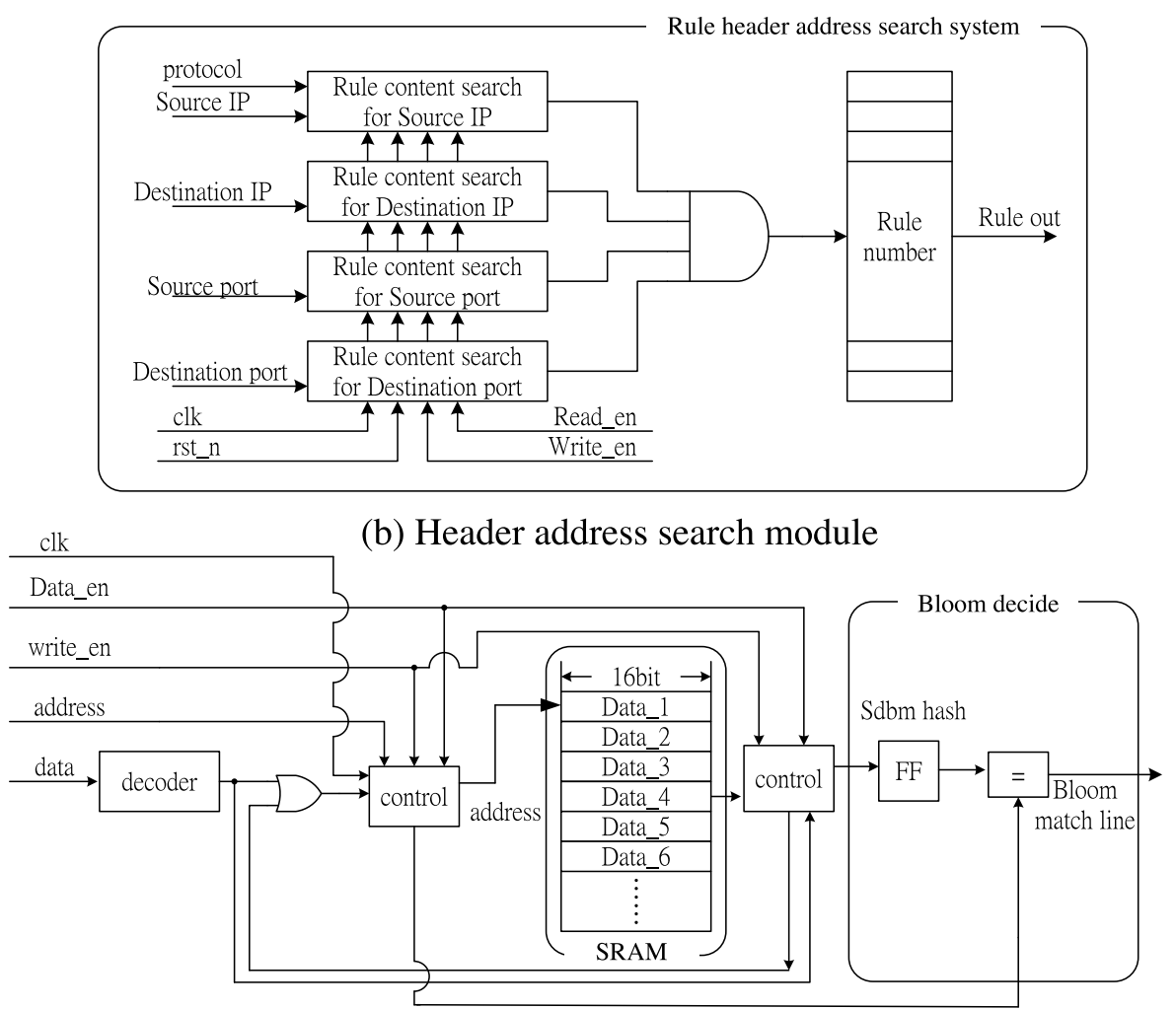

(c) Bloom filter design

Fig. 1. The system architecture of IDS and the submodule designs

The search operation in the IP part is followed by a recorder, the function of which is to count the number of accesses. If the same IP address accesses over a normal access number, then the IP has the potential to spoof the source IP addresses. A general web access for 5 seconds to obtain the same IP is approximately 10 times. Therefore, we defined the denial of service attack. If the same IP accesses more than 20 times in 5 seconds, the system tells the firewall to subsequently block this IP access. The bloom filter design comprised of a hash function and off-chip memory. The architecture of the bloom filter is shown in Fig. 1 (c). The value of the hash function is separated 
is the bits number. When the system is in read mode, the data are read from the memory for the control signal to the bloom decide module. When the information is received, the content is divided into odd and even bytes for byte comparison. According to 8 bits to a cycle of content processing, two parallel sequence comparisons performed a high system throughput, as shown in Fig. 2 (a). The blocks launch a matched or unmatched signal after the comparison is completed. If the received signal is " 0 ," a byte does not match with the rule. These data do not trigger the Snort rules, and the system performs the next parallel comparison. Fig. 2 (b) shows the content compare design.

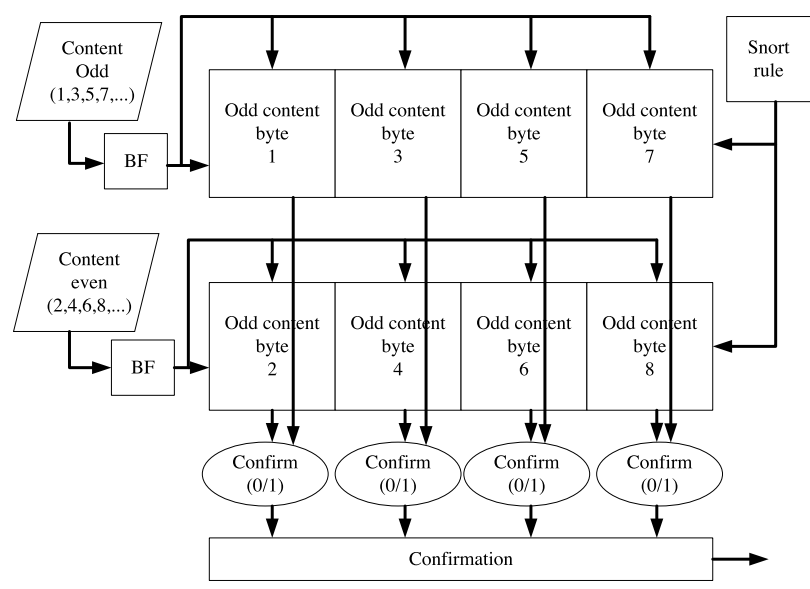

(a) Parallel content compare module

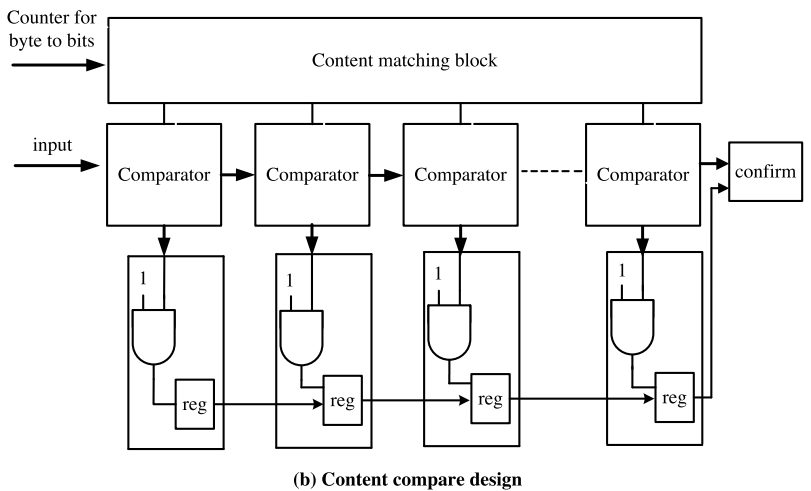

Fig. 2. The content compare module and the content compare design

\section{VLSI implementation and performance analysis}

We implemented the IDS ASIC to develop the high-throughput IDS. The ASIC design was conducted in the Verilog hardware description language and synthesized by Synopsys Design Compiler using the TSMC 0.18 um CMOS single-poly six metals standard cell library. In the chip layout of the IDS system, the area of which is $2.85 * 2.64 \mathrm{~mm}^{2}$. The off-chip memory size is $8 \mathrm{MB}$. The ASIC operated at a maximal operating frequency of $453 \mathrm{MHz}$. Our system is a 16 bits hardware IDS with Snort rules matching. As the 
definition of throughput in the following:

$$
T=\text { clock } \times \text { bits }
$$

Our design achieves 7.2 Gbps system throughputs so that can deal with 1 Gbps requirement of the edge speed on the network. Although the core network speed requirement is over $10 \mathrm{Gbps}$, our design with $7.2 \mathrm{Gbps}$ is near the requirement. The comparison table is shown in Table I. Our system achieves the highest throughput of the comparison in hardware. In the memory design, [1] and [3] used on-chip memory. Our system supports 4,020 snort rules with the off-chip $8 \mathrm{MB}$ memory.

Table I. Comparison results with others works

\begin{tabular}{l|c|c|c|c}
\hline Design & Sourdis [1] & Azimi [2] & Lin [3] & Proposed \\
\hline Implementation & FPGA & ASIC & ASIC & ASIC \\
\hline \# of Snort rules & 3791 & - & 2217 & 4020 \\
\hline Speed (MHz) & 303 & 166 & 330 & 453 \\
\hline $\begin{array}{l}\text { On-chip memory } \\
\text { (MB) }\end{array}$ & 6 & - & 0.15 & - \\
\hline $\begin{array}{l}\text { Off-chip } \\
\text { memory } \\
\text { (MB) }\end{array}$ & - & 10 & - & 8 \\
\hline $\begin{array}{l}\text { Throughput } \\
\text { (Gbps) }\end{array}$ & 2.7 & 2 & 4 & 7.2 \\
\hline
\end{tabular}

\section{Conclusion}

The IDS architectures for virus detection are rapidly developed. We designed and implemented a high-throughput IDS that compares the header and the content part of a packet. The parallel pattern matching uses the odd and even bytes to compare the number of Snort rules. The system also monitors the dedicated IP addresses and then tells the firewall to protect denial of service attack. According to the protocol and payload separation of the parallel comparison, our design performs high system throughput. The IDS ASIC reaches $453 \mathrm{MHz}$ that achieves $7.2 \mathrm{Gbps}$ throughput to deal with the traffic requirement of edge speed in end user network. With $8 \mathrm{MB}$ off-chip SRAM, the system performs intrusion detection for 4,020 Snort rules that the pattern number is enough for intruder signature. 